\title{
Alteration of dominant cyanobacteria in different bloom periods caused by abiotic factors and species interactions
}

\author{
Zhenyan Zhang ${ }^{1}$, Xiaoji Fan ${ }^{2}$, W.J.G.M. Peijnenburg ${ }^{3,4}$, Meng Zhang ${ }^{1}$, \\ Liwei Sun ${ }^{1}$, Yujia Zhai ${ }^{3}$, Qi Yu ${ }^{3}$, Juan $\mathrm{Wu}^{3}$, Tao Lu ${ }^{1}$, Haifeng Qian ${ }^{1, *}$ \\ ${ }^{1}$ College of Environment, Zhejiang University of Technology, Hangzhou 310032, China \\ ${ }^{2}$ College of Biotechnology and Bioengineering, Zhejiang University of Technology, Hangzhou 310032, China \\ ${ }^{3}$ Institute of Environmental Sciences (CML), Leiden University, 2300 RA, Leiden, the Netherlands \\ ${ }^{4}$ National Institute of Public Health and the Environment (RIVM), Center for Safety of Substances and Products, P.O. \\ Box 1, Bilthoven, the Netherlands
}

A R T I C L E I N F O

Article history:

Received 25 March 2020

Revised 27 May 2020

Accepted 3 June 2020

Available online 21 June 2020

Keywords:

Cyanobacterial bloom

Temperature

Nitrogen condition

Species interaction

\begin{abstract}
A B S T R A C T
Freshwater cyanobacterial blooms have drawn public attention because they threaten the safety of water resources and human health worldwide. Heavy cyanobacterial blooms outbreak in Lake Taihu in summer annually and vanish in other months. To find out the factors impacting the cyanobacterial blooms, the present study measured the physicochemical parameters of water and investigated the composition of microbial community using the $16 \mathrm{~S}$ rRNA gene and internal transcribed spacer amplicon sequencing in the months with or without bloom. The most interesting finding is that two major cyanobacteria, Planktothrix and Microcystis, dramatically alternated during a cyanobacterial bloom in 2016, which is less mentioned in previous studies. When the temperature of the water began increasing in July, Planktothrix appeared first and showed as a superior competitor for M. aeruginosa in $\mathrm{NO}_{3}{ }^{-}$-rich conditions. Microcystis became the dominant genus when the water temperature increased further in August. Laboratory experiments confirmed the influence of temperature and the total dissolved nitrogen (TDN) form on the growth of Planktothrix and Microcystis in a co-culture system. Besides, species interactions between cyanobacteria and non-cyanobacterial microorganisms, especially the prokaryotes, also played a key role in the alteration of Planktothrix and Microcystis. The present study exhibited the alteration of two dominant cyanobacteria in the different bloom periods caused by the temperature, TDN forms as well as the species interactions. These results helped the better understanding of cyanobacterial blooms and the factors which contribute to them.
\end{abstract}

(C) 2020 The Research Center for Eco-Environmental Sciences, Chinese Academy of Sciences. Published by Elsevier B.V.

\section{Introduction}

There is growing awareness that cyanobacterial blooms seriously threaten the safety of public water resources. Thus, they have become a widespread environmental problem. The development of a cyanobacterial bloom dramatically influences

\footnotetext{
* Corresponding author.

E-mail: hfqian@zjut.edu.cn (H. Qian).
}

the water quality, notably increases the turbidity of water and disrupts the acid-base equilibrium (Huisman et al., 2018; Paerl and Huisman, 2008). Toxins or other allelochemicals produced by nuisance cyanobacteria strains also pose a potential threat to aquatic microbes (Qian et al., 2018; Rouhiainen et al., 2000; Song et al., 2017; Sukenik et al., 2002), impacting the microbial community during blooms. Most importantly, cyanotoxins produced by several cyanobacterial species can harm human health (Elder et al., 1993).

Anthropogenic-induced eutrophication and the rising temperature can be the main factors influence the cyanobacterial blooms in freshwaters worldwide (Huisman et al., 2018; 
Monchamp et al., 2018; Rigosi et al., 2014), although there are also many other factors like the influx of pollutants including fungicide (Lu et al., 2019) and nanoparticles (Lu et al., 2020a). Early reports tended to identify phosphorus (P) as the primary cause of freshwater cyanobacteria blooms (Schindler et al., 2008), while more recent studies have focused on the function of nitrogen $(\mathrm{N})$ and demonstrated that $\mathrm{N}$ played an equal role as $\mathrm{P}$ in limiting the growth of nuisance algae (Elser et al., 2007; Lewis and Wurtsbaugh, 2008; Lewis et al., 2011). Furthermore, Davis et al. (2015) found that bloom growth responded more frequently to the addition of $\mathrm{N}$ than $\mathrm{P}$, and the enrichment of both $\mathrm{N}$ and $\mathrm{P}$ caused the highest microcystin concentrations in Lake Erie.

Considering the long-term trend of global warming (Sevellec and Drijfhout, 2018) and the governmental control of nutrient inputs to freshwater (Conley et al., 2009), temperature will be the major factor in enhancing cyanobacterial blooms. Elevated temperatures have exacerbated massive cyanobacterial blooms in many aquatic ecosystems, favoring the proliferation and dominance of cyanobacteria, as cyanobacteria grow better than diatoms or green algae at high temperatures (Jöhnk et al., 2008; Paerl and Huisman, 2008; Paerl and Huisman, 2009). For example, Microcystis, which are the most frequent bloom-forming cyanobacteria, grows slowly below $20{ }^{\circ} \mathrm{C}$ but reaches a maximum growth rate at approximately $30{ }^{\circ} \mathrm{C}$ (Jöhnk et al., 2008; Paerl and Paul, 2012). In addition, high temperatures can strengthen the vertical stratification of freshwater. Under these conditions, several cyanobacteria species can float upward to the water surface due to the buoyancy of intracellular gas vesicles (Huisman et al., 2018) and absorb most of the solar radiation (Ibelings et al., 2003), resulting in enhanced dominance. Furthermore, some studies indicated that rising temperatures could induce the increased production of toxins (Berry et al., 2017; Kleinteich et al., 2012), which make cyanobacteria more aggressive in freshwater microbial communities. Just a few studies reported microcystin concentration increases at lower temperatures (Peng et al., 2018).

Some researchers unveiled that cyanobacterial communities are spatially and temporally heterogeneous during blooms, whereas the composition and diversity of a microbial community also vary (Berry et al., 2017; Qian et al., 2017; Tromas et al., 2017). Microcystis can inhibit the growth of other microbes by secreting specific metabolites (Song et al., 2017) or via other unknown ways (Bittencourt-Oliveira et al., 2014; Ma et al., 2015). The appearance of distinct microorganisms that are associated with Microcystis and Anabaena blooms (Louati et al., 2015) imply beneficial interactions between cyanobacteria and the associated bacteria. Additionally, heterotrophic bacteria have shown both positive (Grant et al., 2014) and negative (Demuez et al., 2015) effects on cyanobacterial growth and may be an important biotic factor for the formation and alteration of cyanobacteria blooms by species interaction.

Lake Taihu is a shallow lake with a mean depth of $1.9 \mathrm{~m}$ located in the Yangtze Delta $\left(30^{\circ} 55^{\prime} 40^{\prime \prime}-31^{\circ} 32^{\prime} 58^{\prime \prime} \mathrm{N}\right.$; $119^{\circ} 52^{\prime} 32^{\prime \prime}-$ $\left.120^{\circ} 36^{\prime} 10^{\prime \prime} \mathrm{E}\right)$. The rapid development of industry and agriculture near the Lake Taihu watershed has led to eutrophication, with frequent formation of cyanobacterial blooms in recent decades (Song et al., 2017). To find out the factors which contribute to the formation and temporal alterations of cyanobacterial blooms, we monitored Lake Taihu in both bloom (July, August, and September in 2016) and non-bloom (March and May in 2017) months. In the field work, we measured the physicochemical parameters of water, and determined the composition of prokaryotic and fungi communities in each month using 16S rRNA gene and internal transcribed spacer (ITS) amplicon sequencing, respectively. In the meantime, we carried out several laboratory experiments to confirm the results of the field work. From these studies, we considered both biotic and abiotic factors and we aimed at explaining (i) the temporal changes of cyanobacterial community composition in different bloom periods in Lake Taihu; (ii) the key environmental factors that influenced the cyanobacterial community; (iii) the species interactions between cyanobacteria and other microorganisms (non-cyanobacterial prokaryotes and fungi). Taken together, the results of this study are intended to provide a better understanding of the interactions between environmental factors, cyanobacteria blooms and the composition of the non-cyanobacterial microbial community.

\section{Methods and materials}

\subsection{Sample collection and field data}

Water samples were collected for 5 months (July, August, and September in 2016 and March and May in 2017) at 3 stations (Sites A, B and C) in Meiliang Bay (Appendix A Fig. S1). Meiliang Bay is the main location of cyanobacterial blooms and is located in the northern Lake Taihu (Shen et al., 2003). We defined July, August and September as "bloom months" due to the higher contents of chlorophyll a and microcystins in these months as measured by Feng et al. (2016) and Shen et al. (2003), respectively. Thereupon we collected water samples in March and May as "non-bloom months" to compare with the "bloom months". Twelve liters of surface water were collected at every site from a depth of $0.5 \mathrm{~m}$, and the temperature was measured directly. The samples were then transported to the laboratory, and the $\mathrm{pH}$ was measured with a pH meter (FE20, Mettler Toledo, Switzerland). The water samples were filtered through $0.22 \mu \mathrm{m}$ polycarbonate filters to collect all aquatic microorganisms (Lu et al., 2020b), which were frozen at $-80{ }^{\circ} \mathrm{C}$ for further experiments. The filtered water was collected for measurement of total dissolved nitrogen (TDN), total dissolved phosphorus (TDP), nitrate $\left(\mathrm{NO}_{3}{ }^{-}\right)$and ammonium $\left(\mathrm{NH}_{4}{ }^{+}\right)$. Alkaline persulfate was added to filtered water, and then autoclaved at $121{ }^{\circ} \mathrm{C}$ to digest the samples. After that, $10 \% \mathrm{HCl}$ was added to each sample and the absorbance was measured at $220 \mathrm{~nm}$ and $275 \mathrm{~nm}$ in a $1-\mathrm{cm}$ cuvette to obtain the TDN content (D'Elia et al., 1977). Filtered water was digested with potassium persulfate at $121{ }^{\circ} \mathrm{C}$ firstly to convert all forms of $\mathrm{P}$ to orthophosphate. Then ammonium molybdate and tin (II) chloride were added to the digested samples, and the absorbance of molybdenum blue was measured at $700 \mathrm{~nm}$ in a 1-cm cuvette measured to calculate the TDP content (Goulden and Brooksbank, 1975). Commercial kits (Suzhou Comin Biotechnology, China) were used for the measurements of $\mathrm{NO}_{3}{ }^{-}$and $\mathrm{NH}_{4}{ }^{+}$contents in the water samples in accordance with the manufacturer's specifications. The $\mathrm{NO}_{3}{ }^{-}$present in filtered water reacted with salicylic acid under the strong acid conditions present and produced nitrosalicylic acid, which is yellow at alkaline conditions. The content of $\mathrm{NO}_{3}{ }^{-}$was calculated according to the absorbance of yellow products at $410 \mathrm{~nm}$ as the specifications described. The $\mathrm{NH}_{4}{ }^{+}$present in filtered water reacted with pypocholoride and phenol under the strong alkaline conditions. The content of $\mathrm{NH}_{4}{ }^{+}$was calculated by the absorbance of the indophenol blue at $625 \mathrm{~nm}$ according to the specifications of the commercial kit.

\subsection{DNA extraction and sequencing}

Total DNA was extracted from the frozen samples using a Power Soil DNA Isolation Kit (Biomiga, USA) for analysis of aquatic microorganism abundance and diversity. The DNA concentration and purity were monitored on $1 \%$ agarose gels. Dependent on the concentration measured, the DNA was diluted to $1 \mathrm{ng} / \mu \mathrm{L}$ using sterile water. The V3-V4 region of the 16S rRNA gene was amplified using primers 341F (CCTAYGGGRBGCASCAG) and 806R (GGACTACNNGGGTATCTAAT) with the barcode. And the ITS gene was amplified using 
the primers ITS5-1737F (GGAAGTAAAAGTCGTAACAAGG) and ITS2-2043R (GCTGCGTTCTTCATCGATGC) with the barcode. All PCRs were performed with the Phusion ${ }^{\circ}$ High-Fidelity PCR Master Mix (New England Biolabs, UK). After quantification and qualification, the PCR products were purified with Qiagen Gel Extraction Kit (Qiagen, Germany). The purified amplicons were then sequenced on the Illumina HiSeq2500 platform (Illumina, USA). The raw sequencing data have been submitted to the NCBI Sequence Read Archive (SRA) database with accession numbers SRR8398881 to SRR8398925 (16S) and SRR8491695 to SRR8491739 (ITS).

\subsection{Sequence analysis}

The raw tag filtration was performed according to the QIIME (V1.9.1, http://qiime.org/index.html) quality-controlled process to obtain high-quality tags. Uparse software (Uparse V8.1.1861, http://drive5.com/uparse/) was used for sequence analysis. Sequences with $\geq 97 \%$ similarity were assigned to the same operational taxonomic unit (OTU) as our previous study described (Zhang et al., 2019). Taxonomic annotation was performed using the GreenGene Database (http: //greengenes.lbl.gov/cgi-bin/nphindex.cgi) based on the RDPclassifier (Version 11.4, https://github.com/rdpstaff/RDPTools) algorithm. Alpha diversity was calculated with QIIME (Version 1.9.1). Analysis of the correlation between environmental parameters and cyanobacteria as well as the species interactions between cyanobacteria and non-cyanobacterial microbial communities (including prokaryotic and fungi community) was performed using the free online platform of Majorbio I-Sanger Cloud Platform (www.i-sanger.com) and based on the Spearman's rank correlation coefficients. Co-occurrence networks in the present study were all performed by Gephi (0.9.2).

\subsection{Laboratory experiments}

1.4.1. Analysis of the abundance of two main cyanobacteria species by special gene analysis

To confirm the results of 16S rRNA gene sequencing, we analyzed the abundance of Planktothrix and Microcystis in samples from Lake Taihu by using real-time PCR with special primers according to the methods described by Rudi et al. (1997). The primer pairs (CH-CI) were specific to Microcystis, and the primer pairs (CN-CO) were specific to Planktothrix. Real-time PCR was performed with the protocol in Eppendorf Master Cycler ${ }^{\circledR}$ ep RealPlex4 (Wesseling Berzdorf, Germany) as in our previous report (Ke et al., 2020). All primer pair sequences are provided in Appendix A Table S1.

\subsubsection{Co-culture experiment for Planktothrix agardhii and} Microcystis aeruginosa

The unialgal P. agardhii and M. aeruginosa, common species of Planktothrix and Microcystis, respectively, were purchased from the Institute of Hydrobiology, Chinese Academy of Sciences (Wuhan, China). To confirm whether there were some allelochemicals exchanged between Microcystis and Planktothrix that resulted in the replacement of Planktothrix by Microcystis in August, an indirect co-culture experiment was carried out. For this experiment, P. agardhii and M. aeruginosa were cultured in sterilized BG-11 liquid medium (without soil extract) at $25{ }^{\circ} \mathrm{C}$ under $300 \mu \mathrm{mol} \mathrm{m}{ }^{-2} \mathrm{sec}^{-1}$ light intensity using a $12 \mathrm{hr} / 12 \mathrm{hr}$ light-dark cycle. The initial optical density at $680 \mathrm{~nm}\left(\mathrm{OD}_{680}\right)$ of $P$. agardhii and M. aeruginosa were 0.02 . A permeable dialysis cellulose membrane (pore size $12 \mathrm{kDa}$; Sigma-Aldrich, USA) was present between the two cyanobacteria to ensure that allelochemicals (if present) could be transported, as we intended to verify the interactions between $M$. aeruginosa and Chlorella vulgaris (Song et al., 2017). We measured the contents of chlorophyll a at 2, 4, 6, 8, 12 day according to Zhang et al. (2018) to determine the impact of M. aeruginosa on $P$. agardhii.

A direct co-culture was performed to determine the effects of temperature and TDN form on the alterations of Microcystis and Planktothrix. For this, P. agardhii and M. aeruginosa were mixed for co-culturing at $25{ }^{\circ} \mathrm{C}$ and $30{ }^{\circ} \mathrm{C}$, with the same initial $\mathrm{OD}_{680}$ of 0.01 , which was close to the value for Lake Taihu water. A modified BG-11 liquid medium with $10 \mathrm{mg} / \mathrm{L} \mathrm{N}$ content and $1 \mathrm{mg} / \mathrm{L} \mathrm{P}$ (we chose a concentration that was 10-fold higher than that in Lake Taihu due to the low growth rate of the two cyanobacteria species at the nutrient concentrations in the natural aquatic system) was used for co-culture. In this medium, two forms of $\mathrm{TDN}, \mathrm{NO}_{3}{ }^{-}$and $\mathrm{NH}_{4}{ }^{+}$, were selected to verify the contribution of TDN form to the alteration of Planktothrix and Microcystis. The ratio of $P$. agardhii and $M$. aeruginosa in co-culture medium was determined by the abundance of their specific genes, $\mathrm{CN}-\mathrm{CO}$ and $\mathrm{CH}-\mathrm{CI}$, respectively, using real-time PCR according to the methods described by Rudi et al. (1997).

\subsection{Statistical analyses}

The statistical significance of the data in this study was analyzed by analysis of variance (ANOVA, Two-factor with replication) using the Analysis Tools of Excel (Microsoft Corporation, Redmond, WA, USA). All analyses were performed in triplicate except for the determination of the water temperature at each station, afterwards the standard deviation (SD) was calculated.

\section{Results and discussion}

\subsection{Fluctuations in cyanobacteria community composition within one year}

The results of 16S rRNA gene sequencing showed that the cyanobacterial community composition in the freshwater exhibited temporally dynamic changes. The dominant cyanobacteria in July and August 2016 were Planktothrix and Microcystis, respectively (Fig. 1A), and no other cyanobacteria ranked in the top ten. Planktothrix dominant $7.5 \%$ (site A) to $35.7 \%$ (site C) of the total OTUs in July 2016, while this value drastically decreased to $0.1-0.3 \%$ in August 2016. Microcystis represented less than $0.1 \%$ of the prokaryotic community in July 2016 but increased to $4.4 \%-18.0 \%$ at sites A, B and C (Fig. 1A) in August 2016. Both of these cyanobacteria disappeared in between September 2016 and March 2017 (less than $0.5 \%)$. Interestingly, when the bloom faded out in September 2016, two other cyanobacteria, Synechococcus and Limnothrix, drastically increased from less than $0.5 \%$ in July and August to $4.6 \%-7.4 \%$ and $1.1 \%-1.3 \%$, respectively. In March 2017, no cyanobacteria were observed, while Microcystis at Site B recurred in the aquatic microbial community as a predominant microbe representing $20 \%$ of the community in May (Fig. 1A). At sites A and C in May 2017, the relative abundance of Limnothrix was approximately $2.1 \%$. However, the abundance of Anabaena (now called Dolichospermum) was low in all sampling month (Fig. 1A), while it was reported as the second most dominant cyanobacterial bloom genus in Taihu (Chen et al., 2003).

What caught our attention in the results of the 16S rRNA gene sequencing was that the dominant genus of cyanobacteria dramatically altered from July (Planktothrix) to August (Microcystis), which was less mentioned in the previous studies. To confirm this finding, the abundances of the specific genes of Microcystis ( $\mathrm{CH}-\mathrm{CI})$ and Planktothrix $(\mathrm{CN}-\mathrm{CO})$ were determined by qPCR (Fig. 1B). The abundance of CN-CO increased in July 2016 and 2017 (site A) but remained relatively low in the other samples (Fig. 1B). A high abundance was detected for $\mathrm{CH}-\mathrm{CI}$ in August 2016, and in May 2017 (site B). All these results were 

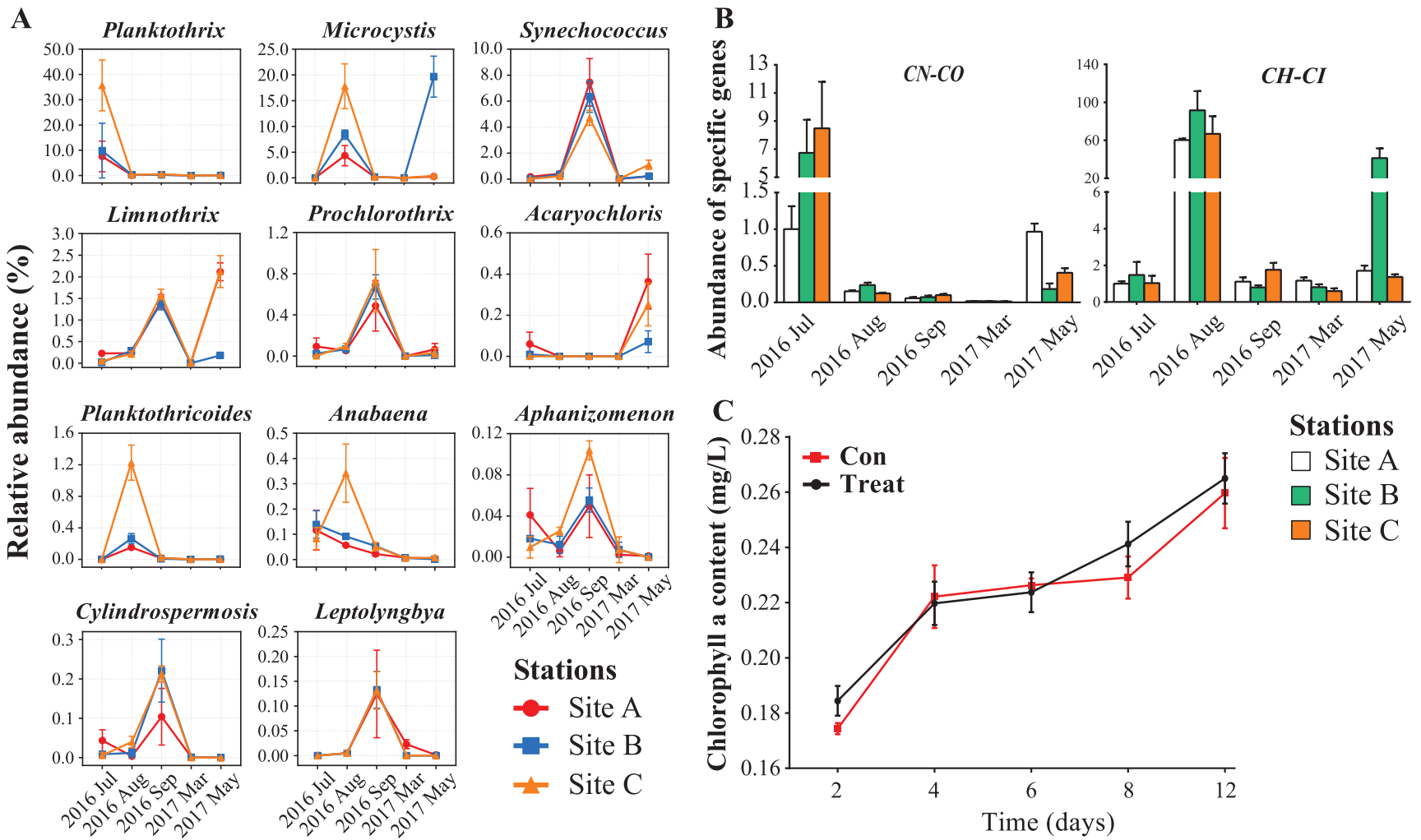

Fig. 1 - Alteration of dominant cyanobacteria in the different bloom periods. (A) The temporally dynamic changes of cyanobacteria. Only the cyanobacteria with relative abundance more than 0.001 at least in one month, are shown; (B) Abundance of Planktothrix (CN-CO) and Microcystis ( $\mathrm{CH}-\mathrm{CI}$ ) specific gene during the different sampling months; (C) The content of chlorophyll a of $P$. agardhii cultured with (treat) or without (control) M. aeruginosa in laboratory experiment.

in accordance with the results of the 16S rRNA gene sequencing. As previous studies have shown, one microbe can inhibit or induce others via the release of specific chemicals, which are called "allelochemicals" (Aharonovich and Sher, 2016; Song et al., 2017). According to this phenomenon, we postulate first that there may be some allelochemicals exchanged between Microcystis and Planktothrix, and then cause the result that Microcystis replaced Planktothrix in August. Therefore, we carried out a laboratory experiment in which we cultured P. agardhii with (treatment) or without (control) M. aeruginosa. However, we did not observe a significant interaction between M. aeruginosa and P. agardhii. The content of chlorophyll a was not influenced by $M$. aeruginosa treatment, compared to the control (Fig. 1C). Thus, the disappearance of Planktothrix and dominance of Microcystis in August 2016 may be mainly attributed to other factors, which include temperature, nutrition condition and the species interaction.

\subsection{Physicochemical parameters of water quality}

The physicochemical water parameters were measured during all sampling months, and included temperature, $\mathrm{pH}$, and the contents of TDN, TDP, $\mathrm{NO}_{3}^{-}$and $\mathrm{NH}_{4}{ }^{+}$. The environmental parameters at the three sampling sites were found to change significantly between sampling months. A maximum water temperature of $28{ }^{\circ} \mathrm{C}$ occurred in August (bloom month), and the minimum temperature was $15{ }^{\circ} \mathrm{C}$ in March (non-bloom month, Table 1) during the sampling period. This result was agreed with the previous studies, which showed that the higher temperature can induce the formation of cyanobacterial bloom (Paerl and Huisman, 2008). The pH varied between 7.6 and 8.9 during the sampling months, but it was higher in bloom months than in non-bloom months, es-
Table 1 - Physicochemical parameters of Lake Taihu water across spatial and temporal scales.

\begin{tabular}{|c|c|c|c|c|c|c|}
\hline & \multicolumn{2}{|c|}{ Temperature } & \multirow{2}{*}{$\begin{array}{l}\text { TDN } \\
(\mathrm{mg} / \mathrm{L})\end{array}$} & \multirow{2}{*}{$\begin{array}{l}\text { TDP } \\
(\mathrm{mg} / \mathrm{L})\end{array}$} & \multirow{2}{*}{$\begin{array}{l}\mathrm{NO}_{3}^{-} \\
(\mathrm{mg} / \mathrm{L})\end{array}$} & \multirow{2}{*}{$\begin{array}{l}\mathrm{NH}_{4}^{+} \\
(\mathrm{mg} / \mathrm{L})\end{array}$} \\
\hline & $\left({ }^{\circ} \mathrm{C}\right)$ & $\mathrm{pH}$ & & & & \\
\hline 2016 Jul_A & 25.0 & 7.8 & 0.31 & 0.09 & 0.21 & 0.03 \\
\hline 2016 Jul_B & 25.0 & 8.2 & 0.26 & 0.13 & 0.11 & $*$ \\
\hline 2016 Jul_C & 25.0 & 8.4 & 0.64 & 0.11 & 0.11 & 0.20 \\
\hline 2016 Aug_A & 28.0 & 8.3 & 0.47 & 0.17 & 0.07 & 0.09 \\
\hline 2016 Aug_B & 28.0 & 8.5 & 1.04 & 0.16 & 0.09 & 0.11 \\
\hline 2016 Aug_C & 28.0 & 8.9 & 1.95 & 0.18 & 0.09 & 0.44 \\
\hline 2016 Sep_A & 22.0 & 7.8 & 0.63 & 0.13 & 0.26 & 0.32 \\
\hline 2016 Sep_B & 22.0 & 7.8 & 0.62 & 0.11 & 0.26 & 0.18 \\
\hline 2016 Sep_C & 22.0 & 7.9 & 0.86 & 0.12 & 0.24 & 0.24 \\
\hline 2017 Mar_A & 15.0 & 7.7 & 1.21 & 0.13 & 0.20 & 0.19 \\
\hline 2017 Mar_B & 15.0 & 7.7 & 1.58 & 0.11 & 0.14 & 0.15 \\
\hline 2017 Mar_C & 15.0 & 7.8 & 1.25 & 0.12 & 0.12 & 0.13 \\
\hline 2017 May_A & 22.0 & 7.9 & 1.22 & 0.11 & 0.14 & 0.09 \\
\hline 2017 May_B & 22.0 & 8.0 & 1.43 & 0.13 & 0.09 & 0.17 \\
\hline 2017 May_C & 22.0 & 8.1 & 1.37 & 0.12 & 0.13 & 0.19 \\
\hline
\end{tabular}

*: missing data; TDN: total dissolved nitrogen; TDP: total dissolved phosphorus.

pecially in August (8.30-8.86, Table 1). The elevation of $\mathrm{pH}$ could be attributed to the carbon concentrating mechanisms by which $\mathrm{CO}_{2}$ is assimilated in the water by cyanobacteria (Sandrini et al., 2016). TDN was lowest in July (0.26-0.64 mg/L) and highest in August (1.949 mg/L at site C, Table 1). But in general, the TDN content was much higher in the non-bloom months than in the bloom months. The decreased contents 

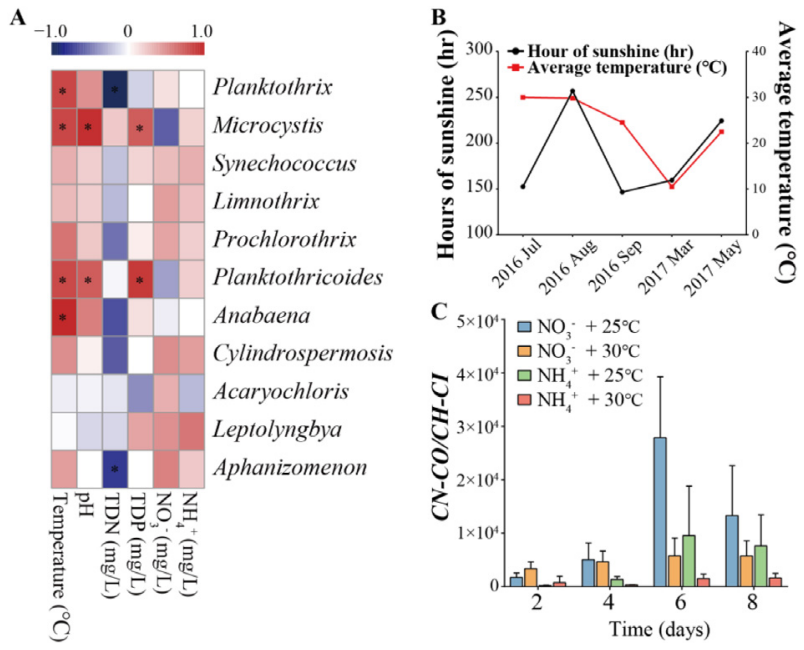

Fig. 2 - Environmental factors related to cyanobacterial community composition. (A) The correlation between environmental parameters and cyanobacterial community composition during the different sampling months. * represent significant correlation ( $p<0.05$ ); (B) Total number of hours of sunshine and average temperature in the sampling months. The data have been provided by the weather bureau of Wuxi, Jiangsu. The black solid line with circles represents the total number of hours of sunshine; the red solid line with squares represents the average temperature; (C) The impacts of temperature and TDN form on the ratio of $\mathrm{P}$. agardhii $(\mathrm{CN}-\mathrm{CO})$ and $\mathrm{M}$. aeruginosa $(\mathrm{CH}-\mathrm{CI})$ specific gene abundance in laboratory experiment. (For interpretation of the references to color in this figure legend, the reader is referred to the web version of this article.)

of TDN in months of bloom was attributed to N-assimilation by cyanobacteria, which is the main uptake mechanism of TDN in the aquatic environment (Salk et al., 2018). The TDN contents increased in non-bloom months due to regeneration (Hampel et al., 2019). Compared to the months with no bloom, the TDP content in the bloom months, except August, did not show any significant differences (Table 1). Xie et al. (2003) indicated that Microcystis blooms in August enhanced the release of TDP from sediment to lake water, and this process is mediated by high pH. Both TDP and $\mathrm{pH}$ reached a peak in August in the present study and this was consistent with the findings of Xie et al. (2003). When focusing on the two TDN forms in July and August, the contents of $\mathrm{NO}_{3}{ }^{-}$were decreased from July to August, while $\mathrm{NH}_{4}{ }^{+}$showed the opposite trend. This finding was similar with the alteration of Microcystis and Planktothrix, which implied that different cyanobacteria may benefit from different TDN forms.

\subsection{Environmental factors related to cyanobacterial community composition}

To determine the correlation between different environmental factors and the cyanobacterial community, we calculated the Spearman's rank correlation coefficient based on the abundances of different genera of cyanobacteria and environmental parameters (Fig. 2A). We observed that Microcystis abundance exhibited a strong positive correlation with $\mathrm{pH}$ (Fig. 2A). This confirms that an increase in cyanobacteria, especially Microcystis, caused an elevation of the $\mathrm{pH}$ of the water due to cyanobacterial carbon concentrating mechanisms (Paerl and Huisman, 2009). Our results also revealed that temperature plays a critical role in the cyanobacterial community, as most listed cyanobacteria were positively correlated with temperature, such as Microcystis and Planktothrix (Fig. 2A). Due to continuous global warming, increasing temperatures dramatically induce the growth of most of cyanobacteria (YvonDurocher et al., 2015). The optimum growth temperature for most of the cyanobacteria is above $25{ }^{\circ} \mathrm{C}$ (Jöhnk et al., 2008) and is different between cyanobacteria species (Robarts and Zohary, 1987). Besides, in the present study, different temperature also played a key role in the alteration of dominant cyanobacteria in different bloom periods. When the temperature of the water increased to $25^{\circ} \mathrm{C}$ in July, Planktothrix appeared firstly (Fig. 1A). Then, Microcystis became the dominant species when the water temperature increased further in $\mathrm{Au}$ gust $2016\left(28{ }^{\circ} \mathrm{C}\right.$, Fig. 1A). A warming event in May 2017 also induced the proliferation of Microcystis (Fig. 1A). It is however unexpected that only site B suffered a Microcystis bloom in May 2017, as the temperature is the same as at sites $\mathrm{A}$ and $\mathrm{C}\left(22^{\circ} \mathrm{C}\right)$. Thus, there must be other factors that influenced the formation of cyanobacteria. Most genera of cyanobacteria have a negative correlation with ambient TDN content, for example, Planktothrix. Microcystis, another common bloom-forming cyanobacterium which was dominated in August, were on the other hand found to be positively correlated with ambient TDN concentration (Fig. 2A). In addition, we found that different forms of TDN influenced the presence of Microcystis and Planktothrix differently: Microcystis were positively correlated with $\mathrm{NH}_{4}{ }^{+}$, while $\mathrm{NO}_{3}{ }^{-}$was beneficial to the growth of Planktothrix (Fig. 2A). Besides, the TDN content and ratio of $\mathrm{NH}_{4}{ }^{+} /$ $\mathrm{NO}_{3}{ }^{-}$was higher in site $\mathrm{B}$ than at the other two sites in May 2017. This could partly explain why only site B suffered a Microcystis bloom in May 2017 at the same temperature. P has been recognized as the primary limiting nutrient of eutrophication (Carpenter, 2008; Schindler et al., 2008), and it positively impacted Microcystis while showing a weakly negative correlation with Planktothrix (Fig. 2A). Apart from the physicochemical water parameters we measured, light is another important factor that can influence cyanobacterial growth. We obtained the data on sunshine time for each sampling month from the weather bureau of Wuxi, Jiangsu (http://js.cma.gov.cn/dsjwz/ wxs/). These data showed that the total number of hours of sunshine in July 2016 was less than in August 2016 (Fig. 2B). This caused Planktothrix to grow better than Microcystis in July 2016, as this species has a high ability to adapt to reduced diel irradiance (Monchamp et al., 2018).

\subsection{Temperature and TDN form influence the growth of $\mathrm{M}$. aeruginosa and $\mathrm{P}$. agardhii in the laboratory}

As shown by the Spearman's rank correlation coefficients, the rising temperature and two forms of TDN might contribute to the alteration of Planktothrix and Microcystis from July to August. To confirm this, we cultured M. aeruginosa and $P$. agardhii together under different conditions (two temperatures and two forms of TDN). The ratio between $P$. agardhii and $M$. aeruginosa specific gene abundance was much higher in the medium containing $\mathrm{NO}_{3}{ }^{-}$than in the medium containing $\mathrm{NH}_{4}{ }^{+}$(Fig. 2C). This result indicated that P. agardhii was a superior competitor for $\mathrm{M}$. aeruginosa in $\mathrm{NO}_{3}{ }^{-}$-rich conditions, a TDN form that is less bioavailable to cyanobacteria than $\mathrm{NH}_{4}{ }^{+}$(McCarthy et al., 2009). It could explain why Planktothrix was dominated in the July (a month with higher $\mathrm{NO}_{3}{ }^{-} / \mathrm{NH}_{4}{ }^{+}$ than August). On the other hand, higher temperatures accelerated the proliferation of M. aeruginosa (Fig. 2C) also explained the replacement of Planktothrix with Microcystis in August (a month with higher temperature). These effects of the two forms of TDN and temperature on the growth of the two cyanobacteria species in the laboratory experiment were consistent with the results of the Spearman's rank correlation coefficients (Fig. 2A). This result indicated that the rising temperature and nutrient condition not only caused the formation of a cyanobacterial bloom as some previous studies showed 
Table 2 - Alpha diversity indices (Shannon, Simpson and Observed species) of prokaryotic and eukaryotic community in samples from different sites and months. The data are presented as mean $\pm \mathrm{SD} . n=3$. Years of each sampling months were the same of

\begin{tabular}{|c|c|c|c|c|c|c|}
\hline & Shannon & Simpson & $\begin{array}{l}\text { Observed } \\
\text { species }\end{array}$ & Shannon & Simpson & $\begin{array}{l}\text { Observed } \\
\text { species }\end{array}$ \\
\hline & $16 S$ & & & ITS & & \\
\hline Jul_A & $5.13 \pm 0.62$ & $0.87 \pm 0.051$ & $933 \pm 235$ & $5.86 \pm 0.56$ & $0.92 \pm 0.04$ & $989 \pm 61$ \\
\hline Jul_B & $4.91 \pm 0.92$ & $0.87 \pm 0.046$ & $974 \pm 321$ & $5.54 \pm 0.48$ & $0.90 \pm 0.04$ & $945 \pm 74$ \\
\hline Jul_C & $4.14 \pm 0.18$ & $0.83 \pm 0.037$ & $630 \pm 111$ & $5.45 \pm 0.61$ & $0.91 \pm 0.02$ & $786 \pm 262$ \\
\hline Aug_A & $7.65 \pm 0.10$ & $0.98 \pm 0.001$ & $1481 \pm 54$ & $4.68 \pm 0.33$ & $0.88 \pm 0.03$ & $758 \pm 34$ \\
\hline Aug_B & $7.48 \pm 0.15$ & $0.98 \pm 0.002$ & $1396 \pm 104$ & $4.80 \pm 0.29$ & $0.88 \pm 0.01$ & $737 \pm 160$ \\
\hline Aug_C & $6.27 \pm 0.28$ & $0.95 \pm 0.013$ & $1074 \pm 133$ & $5.22 \pm 0.29$ & $0.92 \pm 0.01$ & $761 \pm 109$ \\
\hline Sep_A & $6.98 \pm 0.35$ & $0.96 \pm 0.004$ & $1592 \pm 299$ & $5.00 \pm 0.55$ & $0.90 \pm 0.02$ & $773 \pm 176$ \\
\hline Sep_B & $7.72 \pm 0.19$ & $0.98 \pm 0.002$ & $1966 \pm 101$ & $4.96 \pm 0.48$ & $0.89 \pm 0.03$ & $846 \pm 160$ \\
\hline Sep_C & $6.93 \pm 0.26$ & $0.96 \pm 0.004$ & $1637 \pm 149$ & $4.46 \pm 0.56$ & $0.87 \pm 0.04$ & $665 \pm 216$ \\
\hline Mar_A & $5.22 \pm 0.10$ & $0.92 \pm 0.007$ & $655 \pm 56$ & $5.21 \pm 1.44$ & $0.89 \pm 0.13$ & $504 \pm 93$ \\
\hline Mar_B & $4.37 \pm 0.19$ & $0.77 \pm 0.023$ & $686 \pm 42$ & $5.07 \pm 0.32$ & $0.91 \pm 0.01$ & $433 \pm 26$ \\
\hline Mar_C & $5.06 \pm 0.30$ & $0.89 \pm 0.047$ & $637 \pm 71$ & $5.12 \pm 1.13$ & $0.93 \pm 0.05$ & $435 \pm 122$ \\
\hline May_A & $4.79 \pm 0.13$ & $0.86 \pm 0.002$ & $733 \pm 31$ & $3.30 \pm 0.48$ & $0.74 \pm 0.07$ & $320 \pm 52$ \\
\hline May_B & $5.12 \pm 0.19$ & $0.89 \pm 0.019$ & $784 \pm 45$ & $3.02 \pm 1.49$ & $0.67 \pm 0.32$ & $291 \pm 69$ \\
\hline May_C & $6.71 \pm 0.24$ & $0.97 \pm 0.006$ & $1028 \pm 89$ & $4.87 \pm 0.11$ & $0.90 \pm 0.01$ & $500 \pm 54$ \\
\hline
\end{tabular}

(Lewis et al., 2011; Paerl and Huisman, 2008), but also played a critical role in the alternation of dominant cyanobacteria in different bloom periods. The results of ANOVA also showed temperature effected more significantly on the growth of the two cyanobacteria species than TDN form (Appendix A Table S2). However, the laboratory experiments didn't show the combined effect of temperature and $\mathrm{N}$ form on the growth of the two cyanobacteria species. This was different with the results of Wang et al. (2016), which found that nutrient levels effected the sensitivities of biodiversity to temperature change. This is because we focused on only two of the main cyanobacteria species rather than on the whole ecosystem, the results of ecosystem would be more complex because of the species interactions.

\subsection{The interaction of the non-cyanobacterial prokaryotic community with cyanobacteria}

The prokaryotic community in months of cyanobacterial bloom (July, August and September) had a higher diversity (Shannon and Simpson index) and richness (observed species) than in non-bloom months (March and May) (Table 2). This result was consistent with the report of Song et al. (2016), which showed that both diversity indices and the richness of the microorganism community increased simultaneously after cyanobacterial blooms occurred in summer and autumn in the West Lake. Besides, during the bloom months, the diversity and richness of the prokaryotic community were relatively low in the early stage of a bloom (July, dominated by Planktothrix) but increased during the development (August, dominated by Microcystis) and decay (September) stages (Table 2). This result showed that the alteration of the dominant genus could also affect the diversity and richness of prokaryotic community in the different cyanobacterial bloom periods.

In detail, the prokaryotic community composition in freshwater exhibited different temporal dynamics (Fig. 3A). The change in abundance of some non-cyanobacterial prokaryotes evoked our interest, as these species showed both positive and negative interactions with the occurrence of a cyanobacterial bloom (Appendix A Fig. S2). Co-occurrence analysis clearly showed that different cyanobacteria were related to different non-cyanobacterial prokaryotic communities. This result implied that the interactions between non-cyanobacterial prokaryote and cyanobacteria could influence the alteration of the dominant genus in different cyanobacterial bloom periods (Appendix A Fig. S2). To clarify this in detail, the top 20 genera of prokaryotic community were chosen for further analysis (Fig. 3B). Roseomonas was positively correlated with Microcystis (Fig. 3B) and co-occurred in August 2016 and May 2017 (Fig. 3A). Relative abundances of Roseomonas in August 2016 increased from site A (1.44\%, respectively) to C $(2.82 \%$, respectively), which corresponded to the changes in Microcystis. Two genera of bacteria affiliated with Pseudomonadales (Pseudomonas and Acinetobacter) and one genus of Aeromonadales (Aeromonas) reached relatively high abundances in July 2016 when Planktothrix bloomed and vanished in other sampling months, clearly showing the positive correlation with Planktothrix (Fig. 3). Two genera of bacteria affiliated with Actinobacteria, hgcI_clade and the L500-29_marine_group, were abundant and constituted approximately $0.40-18.37 \%$ and $0.70-7.53 \%$ of all species throughout the year, respectively (Fig. 3A). Though these two Actinobacteria did not show either a positive or a negative correlation with Microcystis or Planktothrix in the entire year, their correlations showed more complex. During the Microcystis outbreak in August 2016, the hgcI_clade and the L50029_marine_group proliferated to high abundances $(6.19 \%$ and $3.75 \%$, respectively). However, once these two genera of Actinobacteria became the dominant species, the abundance of Microcystis decreased until May 2017 (Fig. 1).

Similar to the feedback interactions between plant and rhizosphere microorganisms (Lu et al., 2018; Qu et al., 2020), there are complex interactions between phycosphere microorganisms and algae (Amin et al., 2015). The most popular connection between bacteria and algae is the exchange of substances. In some conditions, heterotrophic bacteria can benefit from algae (such as provision of carbon sources) and provide the substances needed by algae in return (such as $\mathrm{VB}_{12}$ ), which is a positive interaction (Grant et al., 2014; Kazamia et al., 2012). The simultaneous variation between Microcystis and non-cyanobacteria like Roseomonas (Fig. 3B) might imply the presence of potential interactions between them, from which both species can benefit. There are also some negative interactions, as bacteria can disrupt the cell envelope and lyse algae rapidly by releasing algicidal agents (Demuez et al., 2015). The three genera (Pseudomonas, Acinetobacter and Aeromonas) which showed positive correlation with Planktothrix are widespread in freshwater (Cai et al., 2014; Scherer et al., 2017), and some of them exhibit species-specific 
A

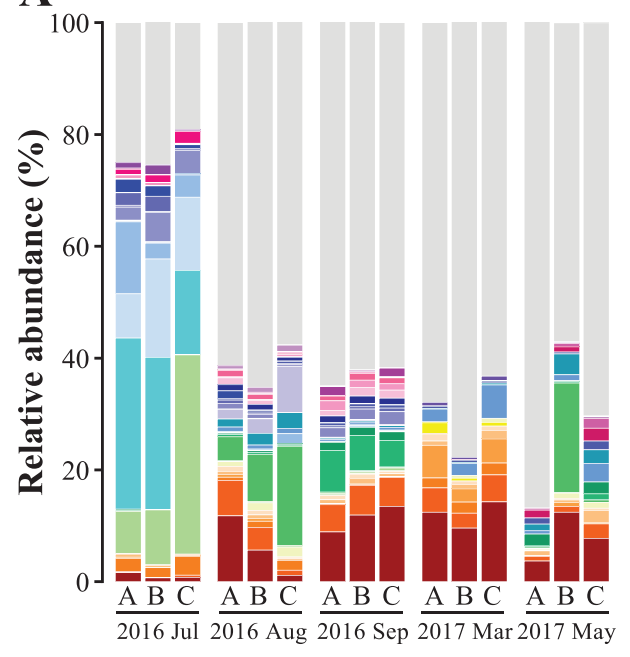

B

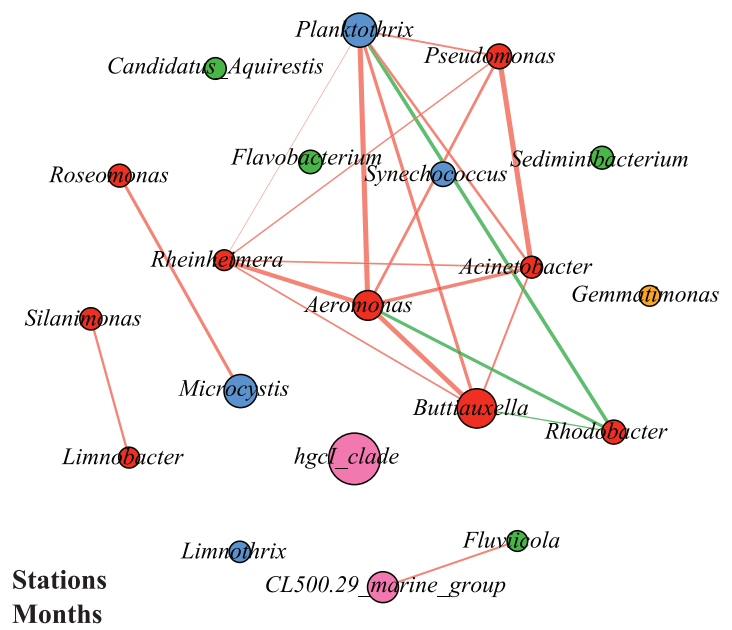

Phylum

Cyanobacteria

Proteobacteria

Bacteroidetes

Actinobacteria

Gemmatimonadetes

$\begin{array}{ccl}\text { Gemmatimonadetes } & \text { Proteobacteria } & \\ \text { Gemmatimonas } & \text { Buttiauxella } & \text { OM27 clade } \\ & \text { Aeromonas } & \text { Vogesella } \\ & \text { Pseudomonas } & \text { BD1-7_clade } \\ \text { Cyanobacteria } & \text { Rhodobacter } & \text { MWH-UniPl_aquatic_group } \\ \text { Planktothrix } & \text { Roseomonas } & \text { Dechloromonas } \\ \text { Microcystis } & \text { Silanimonas } & \text { Acidibacter } \\ \text { Synechococcus } & \text { Acinetobacter } & \text { Paucibacter } \\ \text { Limnothrix } & \text { Limnobacter } & \text { Rickettsia } \\ & \text { Rheinheimera } & \text { Brevundimonas }\end{array}$

Proteobacteria
Buttiauxella
Aeromonas
Pseudomonas
Rhodobacter
Roseomonas
Silanimonas
Acinetobacter
Limnobacter
Rheinheimera Brevundimonas

Fig. 3 - Composition of the prokaryotic microbial community during the different sampling months and the correlation between cyanobacteria and non-cyanobacterial prokaryotes. (A) The top 10 genera in the prokaryotic microbial community composition in each sampling months. Considering the top 10 genera changed in different months significantly, 34 genera were sorted as "top 10 genera" of specific months and drawn together; (B) Co-occurrence analysis of the top 20 genera in the prokaryotic microbial community. Size and color of the nodes represent the relative abundance and phylum of the genera, respectively. Lines in red and green denote positive and negative correlations, respectively. The width reflects the strength of the correlation. Only showed strong (Spearman's $r>0.8$ or $r<-0.8)$ and significant $(p<0.05)$ correlation. (For interpretation of the references to color in this figure legend, the reader is referred to the web version of this article.)

algicidal activity and can lysis cyanobacteria in particular (Scherer et al., 2017). For example, Aeromonas can lyse Microcystis (Yang et al., 2013), which may partly explain why the abundance of Microcystis was low in July. Thus, we postulate that Planktothrix could obtain a dominant status in cyanobacteria by inducing some algicidal bacteria to inhibit Microcystis in July. However, in some cases, the interactions between bacteria and algae are more complex. For example, bacteria and algae mutually benefited from each other firstly. However, when bacteria became dominant, they secreted excess substances that are beneficial to algae at low concentrations but toxic at high concentrations (Segev et al., 2016). For example, Actinobacteria was one of the most common taxa in freshwater and showed a different correlation to cyanobacterial blooms at the subclade level (Berry et al., 2017). The relationships of the hgcI_clade and the L500-29_marine_group with Microcystis were similar to those reported by Segev et al. (2016), implying that they benefited from Microcystis in the preliminary growth stage but returned toxicants, which in turn inhibited the proliferation of Microcystis. Above all, considering the various and complex interactions between bacteria and algae, bacteria in the freshwater environment in this study may have had a biotic effect on cyanobacterial proliferation and alteration in similar ways.

\subsection{The interaction of the fungi community with cyanobacteria}

The ITS sequence was analyzed to clarify the fungi community composition (Fig. 4) and diversity (Table 2). Similar to the prokaryotic microbial community, the fungi community showed higher diversity and richness in bloom months than in non-bloom months (Table 2). However, the three alpha diversity indices did not change significantly. In the fungi community, Bullera was observed in all of the sampling months and sites, and this species dominated in nearly all months with proportions greater than 50\% except in March 2017, which was a cold month $(3.4 \%$ to $11.9 \%$ at the three sampling sites, Fig. 4A). As Bullera occupied most taxa and other genera of fungi were always relatively rare, cyanobacteria did not show a significant correlation with the fungi community (Appendix A Fig. S3). There were 397 genera with abundances less than $1 \%$ in all samples, and less than $0.01 \%$ in several samples. These genera were defined as rare genera according to Xue et al. (2018). The rare fungi community composition exhibited different temporal and spatial dynamics (Fig. 4B). This result indicated that the rare fungi community was more sensitive to cyanobacterial blooms than the abundant community. Xue et al. (2018) also showed that a cyanobacterial bloom in a reservoir impacted rare eukaryotic plankton communities more dramatically than abundant microbial communities. Although the abundance of the rare fungi community was low, it was the keystone of the aquatic environment (Jousset et al., 2017). Many species with rare abundance play an important role in regulating the functions of the environment, and they may regulate nutrient cycling and indirectly interact with the abundant microbial community (Jousset et al., 2017). Above all, cyanobacteria did not significantly alter the fungi community diversity but changed the compo- 

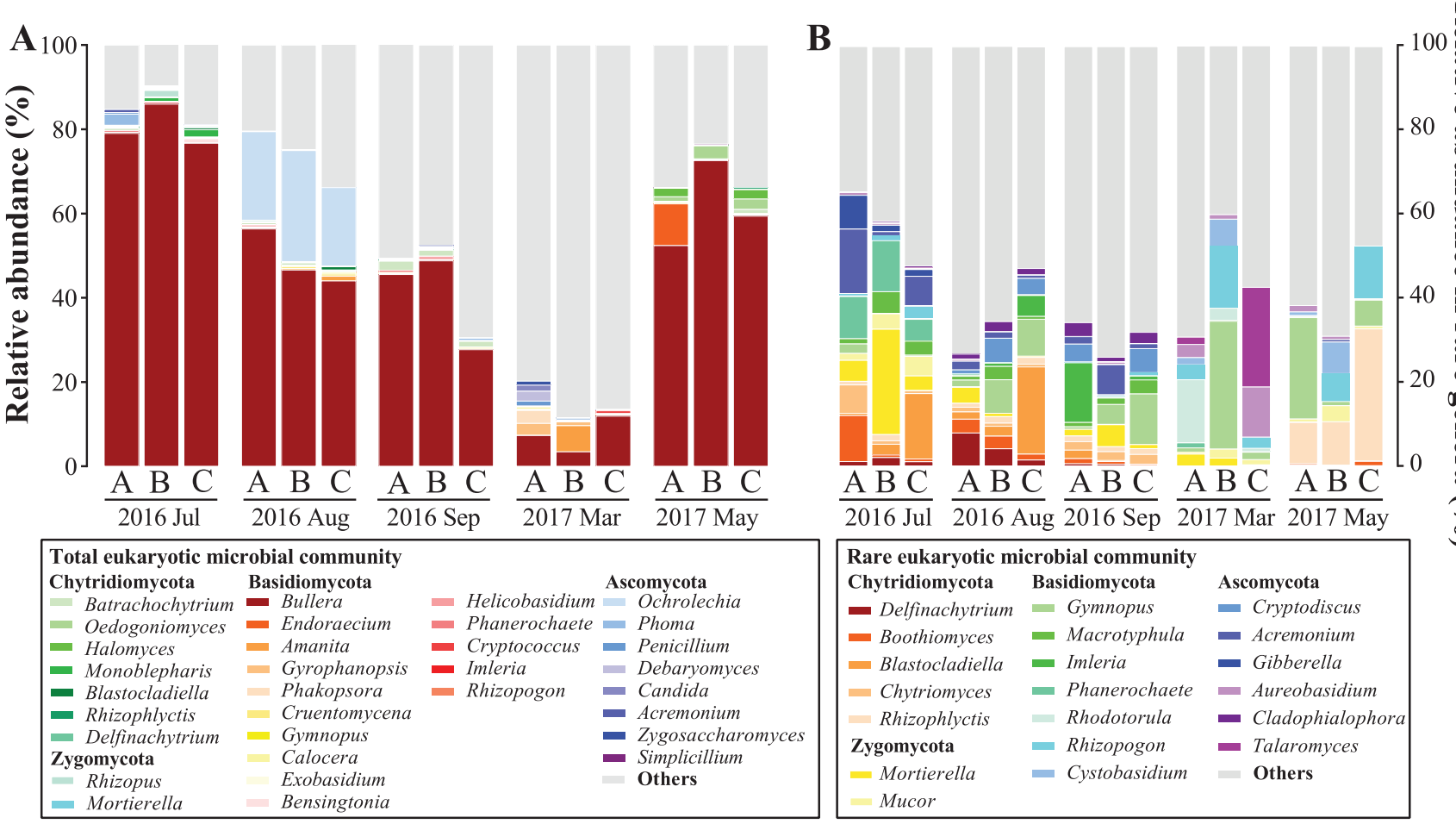



Fig. 4 - Changes in the fungi community composition in sampling months. (A) The top 10 genera in the fungi community composition in each sampling month. Considering the top 10 genera changed in different months, 32 genera were sorted as "top 10 genera" of specific months and drawn together; (B) The top 20 genera (sorted as the total relative abundance in all samples) in rare fungi community composition during the different sampling months.

sition of the rare fungi community dramatically. Besides, compared to the non-cyanobacterial prokaryotic community, the fungi community showed weaker interactions to the cyanobacteria.

\section{Conclusions}

Our results revealed that the microbial community composition in Lake Taihu exhibited temporally dynamic changes, along with the change of environmental factors. More interestingly, the presence of the dominant cyanobacteria altered in different bloom months, as Planktothrix and Microcystis dominated in July and August, respectively. It was confirmed that there is no allelochemical exchanged between M. aeruginosa and P. agardhii growth, which are common species of Planktothrix and Microcystis, respectively. The combined results of field work and laboratory experiments showed that the temperature and the TDN form were the main abiotic factors which contributed to the alternation of Planktothrix and Microcystis from July to August. Planktothrix showed as a superior competitor for Microcystis in $\mathrm{NO}_{3}{ }^{-}$-rich conditions and became dominant in July, while Microcystis was dominant in August along with the rising temperature. Additionally, different dominant cyanobacteria showed different patterns of microbial community. As a result of species interactions, the microbial community in the phycosphere, especially the noncyanobacterial prokaryotic community, also contributed to the alternation of cyanobacteria. In conclusion, the present study exhibited that two dominant cyanobacteria dramatically altered in different bloom periods in Lake Taihu due to the contribution of temperature, nutrient conditions and species interactions. As most of the previous studies only focused on the formation of cyanobacterial blooms and its reason, these re- sults provide a new understanding of cyanobacterial blooms. However, there are still some interesting studies which need to be performed in the future. It is worth mentioning that the causal relationship between cyanobacteria alternation and microbial community assemblage needs to be confirmed in further studies under ingenious design.

\section{Acknowledgments}

This work was supported by National Natural Science Foundation of China (Nos. 21577128, 21777144), and the Program for Changjiang Scholars and Innovative Research Team in University (No. IRT17R97).

\section{Appendix A. Supplementary data}

Supplementary material associated with this article can be found, in the online version, at doi:10.1016/j.jes.2020.06.001.

\section{REFERENCES}

Aharonovich, D., Sher, D., 2016. Transcriptional response of Prochlorococcus to co-culture with a marine Alteromonas: differences between strains and the involvement of putative infochemicals. ISME J. 10, 2892-2906.

Amin, S.A., Hmelo, L.R., van Tol, H.M., Durham, B.P., Carlson, L.T., Heal, K.R., et al., 2015. Interaction and signalling between a cosmopolitan phytoplankton and associated bacteria. Nature 522, 98-101.

Berry, M.A., Davis, T.W., Cory, R.M., Duhaime, M.B., Johengen, T.H., Kling, G.W., et al., 2017. Cyanobacterial harmful algal blooms are a biological disturbance to Western Lake Erie bacterial communities. Environ. Microbiol. 19, 1149-1162.

Bittencourt-Oliveira, M.D.C., Chia, M.A., de Oliveira, H.S.B., Cordeiro Araújo, M.K., Molica, R.J.R., Dias, C.T.S., 2014. Allelopathic interactions between microcystin-producing and non-microcystin-producing cyanobacteria and green microalgae: implications for microcystins production. J. Appl. Phycol. 27, 275-284. 
Cai, H., Jiang, H., Krumholz, L.R., Yang, Z., 2014. Bacterial community composition of size-fractioned aggregates within the phycosphere of cyanobacterial blooms in a eutrophic freshwater lake. PLoS ONE 9, e102879.

Carpenter, S.R., 2008. Phosphorus control is critical to mitigating eutrophication. Proc. Natl. Acad. Sci. U. S. A. 105, 11039-11040.

Chen, Y., Qin, B., Teubner, Katrin, Dokulil, M.T., 2003. Long-term dynamics of phytoplankton assemblages: Microcystis-domination in Lake Taihu, a large shallow lake in China. J. Plankton Res. 25, 445-453.

Conley, D.J., Paerl, H.W., Howarth, R.W., Boesch, D.F., Seitzinger, S.P., Havens, K.E., et al. 2009. Controlling eutrophication: nitrogen and phosphorus. Science 323, 1014-1015.

D'Elia, C.F., Steudler, P.A., Corwin, N., 1977. Determination of total nitrogen in aqueous samples using persulfate digestion. Limnol. Oceanogr. 22, 760-764.

Davis, T.W., Bullerjahn, G.S., Tuttle, T., McKay, R.M., Watson, S.B., 2015. Effects of increasing nitrogen and phosphorus concentrations on phytoplankton community growth and toxicity during Planktothrix blooms in Sandusky Bay. Lake Erie. Environ. Sci. Technol. 49, 7197-7207.

Demuez, M., Gonzalez-Fernandez, C., Ballesteros, M., 2015. Algicidal microorganisms and secreted algicides: New tools to induce microalgal cell disruption. Biotechnol. Adv. $33,1615-1625$.

Elder, G.H., Hunter, P.R., Codd, G.A., 1993. Hazardous freshwater cyanobacteria (blue-green algae). Lancet $341,1519-1520$.

Elser, J.J., Bracken, M.E., Cleland, E.E., Gruner, D.S., Harpole, W.S., Hillebrand, H., et al., 2007. Global analysis of nitrogen and phosphorus limitation of primary producers in freshwater, marine and terrestrial ecosystems. Ecol. Lett. 10, 1135-1142.

Feng, L., Liu, S., Wu, W., Ma, J., Li, P., Xu, H., et al., 2016. Dominant genera of cyanobacteria in Lake Taihu and their relationships with environmental factors. J. Microbiol. 54, 468-476.

Goulden, P.D., Brooksbank, P., 1975. The determination of total phosphate in natural waters. Anal. Chim. Acta. 80, 181-187.

Grant, M.A., Kazamia, E., Cicuta, P., Smith, A.G., 2014. Direct exchange of vitamin B12 is demonstrated by modelling the growth dynamics of algal-bacterial cocultures. ISME J. 8, 1418-1427.

Hampel, J.J., McCarthy, M.J., Neudeck, M., Bullerjahn, G.S., McKay, R.M.L., Newell, S.E., 2019 Ammonium recycling supports toxic Planktothrix blooms in Sandusky Bay, Lake Erie: Evidence from stable isotope and metatranscriptome data. Harmful Algae 81, 42-52.

Huisman, J., Codd, G.A., Paerl, H.W., Ibelings, B.W., Verspagen, J.M.H., Visser, P.M., 2018. Cyanobacterial blooms. Nat. Rev. Microbiol. 16, 471-483.

Ibelings, B.W., Vonk, M., Los, H.F.J., Molen, D.T.v.d., Mood, W.M., 2003. Fuzzy modeling of cyanobacterial surface waterblooms: validation with NOAA-AVHRR satellite images. Ecol. Appl. 13, 1456-1472.

Jöhnk, K.D., Huisman, J.E.F., Sharples, J., Sommeijer, B.E.N., Visser, P.M., Stroom, J.M., 2008. Summer heatwaves promote blooms of harmful cyanobacteria. Global Change Biol. 14, 495-512.

Jousset, A., Bienhold, C., Chatzinotas, A., Gallien, L., Gobet, A., Kurm, V., et al., 2017. Where less may be more: how the rare biosphere pulls ecosystems strings. ISME J. 11, 853-862.

Kazamia, E., Czesnick, H., Nguyen, T.T., Croft, M.T., Sherwood, E., Sasso, S., et al., 2012. Mutualistic interactions between vitamin B12-dependent algae and heterotrophic bacteria exhibit regulation. Environ. Microbiol. 14, 1466-1476.

Ke, M., Li, Y., Qu, Q., Ye, Y., Peijnenburg, W., Zhang, Z., et al., 2020. Offspring toxicity of silver nanoparticles to Arabidopsis thaliana flowering and floral development. J. Hazard. Mater. 386, 121975

Kleinteich, J., Wood, S.A., Küpper, F.C., Camacho, A., Quesada, A., Frickey, T., et al., 2012. Temperature-related changes in polar cyanobacterial mat diversity and toxin production. Nat. Clim. Chang. 2, 356-360.

Lewis, W.M., Wurtsbaugh, W.A., 2008. Control of lacustrine phytoplankton by nutrients: erosion of the phosphorus paradigm. Int. Rev. Hydrobiol. 93, 446-465.

Lewis, W.M., Wurtsbaugh, W.A., Paerl, H.W., 2011. Rationale for control of anthropogenic nitrogen and phosphorus to reduce eutrophication of inland waters. Environ. Sci. Technol. 45, 10300-10305.

Louati, I., Pascault, N., Debroas, D., Bernard, C., Humbert, J.F., Leloup, J., 2015. Structural diversity of bacterial communities associated with bloom-forming freshwater cyanobacteria differs according to the cyanobacterial Genus. PLoS ONE 10, e0140614.

Lu, T., Ke, M., Lavoie, M., Jin, Y., Fan, X., Zhang, Z., et al., 2018. Rhizosphere microorganisms can influence the timing of plant flowering. Microbiome 6, 231.

Lu, T., Qu, Q., Lavoie, M., Pan, X., Peijnenburg, W., Zhou, Z., et al., 2020a. Insights into the transcriptional responses of a microbial community to silver nanoparticles in a freshwater microcosm. Environ. Pollut. 258, 113727.

Lu, T., Xu, N., Zhang, Q., Zhang, Z., Debognies, A., Zhou, Z., et al., 2020b. Understanding the influence of glyphosate on the structure and function of freshwater microbial community in a microcosm. Environ. Pollut. 260, 114012.

Lu, T., Zhang, Q., Lavoie, M., Zhu, Y., Ye, Y., Yang, J., et al., 2019. The fungicide azoxystrobin promotes freshwater cyanobacterial dominance through altering competition. Microbiome 7, 128.

Ma, J., Qin, B., Paerl, H.W., Brookes, J.D., Wu, P., Zhou, J., et al., 2015. Green algal over cyanobacterial dominance promoted with nitrogen and phosphorus additions in a mesocosm study at Lake Taihu, China. Environ. Sci. Pollut. Res. 22, 5041-5049.

McCarthy, M.J., James, R.T., Chen, Y., East, T.L., Gardner, W.S., 2009. Nutrient ratios and phytoplankton community structure in the large, shallow, eutrophic, subtropical Lakes Okeechobee (Florida, USA) and Taihu (China). Limnology 10, 215-227.

Monchamp, M.E., Spaak, P., Domaizon, I., Dubois, N., Bouffard, D., Pomati, F., 2018. Homogenization of lake cyanobacterial communities over a century of climate change and eutrophication. Nat. Ecol. Evol. 2, 317-324.

Paerl, H.W., Huisman, J., 2008. Blooms like it hot. Science 320, 57-58.
Paerl, H.W., Huisman, J., 2009. Climate change: a catalyst for global expansion of harmful cyanobacterial blooms. Environ. Microbiol. Rep. 1, 27-37.

Paerl, H.W., Paul, V.J., 2012. Climate change: links to global expansion of harmful cyanobacteria. Water Res. 46, 1349-1363.

Peng, G., Martin, R.M., Dearth, S.P., Sun, X., Boyer, G.L., Campagna, S.R., et al., 2018. Seasonally relevant cool temperatures interact with $\mathrm{N}$ chemistry to increase microcystins produced in lab cultures of Microcystis aeruginosa. NIES-843. Environ. Sci. Technol. 52, 4127-4136.

Qian, H., Lu, T., Song, H., Lavoie, M., Xu, J., Fan, X., et al., 2017. . Spatial variability of cyanobacteria and heterotrophic bacteria in Lake Taihu (China). Bull. Environ. Contam. Toxicol. 99, 380-384.

Qian, H., Xu, J., Lu, T., Zhang, Q., Qu, Q., Yang, Z., et al., 2018. Responses of unicellular alga Chlorella pyrenoidosa to allelochemical linoleic acid. Sci. Total Environ 625, 1415-1422.

Qu, Q., Zhang, Z., Peijnenburg, W., Liu, W., Lu, T., Hu, B., et al., 2020. . Rhizosphere microbiome assembly and its impact on plant growth. J. Agric. Food Chem. 68, 5024-5038.

Rigosi, A., Carey, C.C., Ibelings, B.W., Brookes, J.D., 2014. The interaction between climate warming and eutrophication to promote cyanobacteria is dependent on trophic state and varies among taxa. Limnol. Oceanogr. 59, 99-114.

Robarts, R.D., Zohary, T., 1987. Temperature effects on photosynthetic capacity, respiration, and growth rates of bloom-forming cyanobacteria. New Zeal. J. Mar. Fresh 21, 391-399.

Rouhiainen, L., Paulin, L., Suomalainen, S., Hyytia"inen, H., Buikema, W., Haselkorn, R., et al., 2000. Genes encoding synthetases of cyclic depsipeptides, anabaenopeptilides, in Anabaena strain 90. Mol. Microbiol. 37, 156-167.

Rudi, K., Skulberg, O., Larsen, F., Jakobsen, K., 1997. Strain characterization and classification of oxyphotobacteria in clone cultures on the basis of 16S rRNA sequences from the variable regions V6, V7, and V8. Appl. Environ. Microbiol. 63, 2593-2599.

Salk, K.R., Bullerjahn, G.S., McKay, R.M.L., Chaffin, J.D., Ostrom, N.E., 2018. Nitrogen cycling in Sandusky Bay, Lake Erie: oscillations between strong and weak export and implications for harmful algal blooms. Biogeosciences 15, 2891-2907.

Sandrini, G., Ji, X., Verspagen, J.M., Tann, R.P., Slot, P.C., Luimstra, V.M., et al., 2016. Rapid adaptation of harmful cyanobacteria to rising $\mathrm{CO}_{2}$. Proc. Natl. Acad. Sci. U. S. A. 113, 9315-9320.

Scherer, P.I., Millard, A.D., Miller, A., Schoen, R., Raeder, U., Geist, J., et al., 2017. Temporal dynamics of the microbial community composition with a focus on toxic cyanobacteria and toxin presence during harmful algal blooms in two South German Lakes. Front. Microbiol. 8, 2387.

Schindler, D.W., Hecky, R.E., Findlay, D.L., Stainton, M.P., Parker, B.R., Paterson, M.J., et al., 2008. Eutrophication of lakes cannot be controlled by reducing nitrogen input: results of a 37-year whole-ecosystem experiment. Proc. Natl. Acad. Sci. U. S. A. 105, $11254-11258$

Segev, E., Wyche, T.P., Kim, K.H., Petersen, J., Ellebrandt, C., Vlamakis, H., et al., 2016. Dynamic metabolic exchange governs a marine algal-bacterial interaction. Elife 5.

Sevellec, F., Drijfhout, S.S., 2018. A novel probabilistic forecast system predicting anomalously warm 2018-2022 reinforcing the long-term global warming trend. Nat. Commun. 9, 3024.

Shen, P.P., Shi, Q., Hua, Z.C., Kong, F.X., Wang, Z.G., Zhuang, S.X., et al., 2003. Analysis of microcystins in cyanobacteria blooms and surface water samples from Meiliang Bay, Taihu Lake. Environ. Int. 29, 641-647.

Song, H., Lavoie, M., Fan, X., Tan, H., Liu, G., Xu, P., et al., 2017. Allelopathic interactions of linoleic acid and nitric oxide increase the competitive ability of Microcystis aeruginosa. ISME J. 11, 1865-1876.

Song, H., Xu, J., Lavoie, M., Fan, X., Liu, G., Sun, L., et al., 2016. Biological and chemical factors driving the temporal distribution of cyanobacteria and heterotrophic bacteria in a eutrophic lake (West Lake, China). Appl. Microbiol. Biotechnol. 101, 1685-1696.

Sukenik, A., Eshkol, R., Livne, A., Hadas, O., Rom, M., Tchernov, D., et al., 2002. Inhibition of growth and photosynthesis of the dinoflagellate Peridinium gatunense by Microcystis sp. (cyanobacteria): A novel allelopathic mechanism. Limnol. Oceanogr 47, 1656-1663.

Tromas, N., Fortin, N., Bedrani, L., Terrat, Y., Cardoso, P., Bird, D., et al., 2017. Characterising and predicting cyanobacterial blooms in an 8-year amplicon sequencing time course. ISME J. 11, 1746-1763.

Wang, J., Pan, F., Soininen, J., Heino, J., Shen, J., 2016. Nutrient enrichment modifies temperature-biodiversity relationships in large-scale field experiments. Nat. Commun. 7, 13960

Xie, L.Q., Xie, P., Tang, H.J., 2003. Enhancement of dissolved phosphorus release from sediment to lake water by Microcystis blooms-an enclosure experiment in a hyper-eutrophic, subtropical Chinese lake. Environ. Pollut. 122, 391-399.

Xue, Y., Chen, H., Yang, J.R., Liu, M., Huang, B., Yang, J., 2018. Distinct patterns and processes of abundant and rare eukaryotic plankton communities following a reservoir cyanobacterial bloom. ISME J. 12, 2263-2277.

Yang, F., Li, X., Li, Y., Wei, H., Yu, G., Yin, L., et al., 2013. Lysing activity of an indigenous algicidal bacterium Aeromonas sp. against Microcystis spp. isolated from Lake Taihu. Environ. Technol. 34, 1421-1427.

Yvon-Durocher, G., Allen, A.P., Cellamare, M., Dossena, M., Gaston, K.J., Leitao, M., et al., 2015. . Five years of experimental warming increases the biodiversity and productivity of phytoplankton. PLoS. Biol. 13, e1002324.

Zhang, Q., Qu, Q., Lu, T., Ke, M., Zhu, Y., Zhang, M., et al., 2018. The combined toxicity effect of nanoplastics and glyphosate on Microcystis aeruginosa growth. Environ. Pollut. 243, 1106-1112.

Zhang, Q., Zhu, D., Ding, J., Zheng, F., Zhou, S., Lu, T., et al., 2019. The fungicide azoxystrobin perturbs the gut microbiota community and enriches antibiotic resistance genes in Enchytraeus crypticus. Environ. Int. 131, 104965. 